J. Phys. IV France 130 (2005) 75-80

(C) EDP Sciences, Les Ulis

DOI: $10.1051 /$ jp4:2005130005

\title{
Mesures en temps réel par diffusion de neutrons aux petits angles (DNPA) avec un appareil à « flux stoppé » (stopped-flow)
}

\section{Grillo}

\author{
Institut Laue-Langevin, DS/LSS, 6 rue Jules Horowitz, BP. 156, 38042 Grenoble Cedex 9 , \\ France
}

\begin{abstract}
Résumé. La nouvelle génération de diffractomètres à haut flux pour la diffusion de neutrons aux petits angles, comme D22 à l'ILL, ouvre de nouvelles perspectives avec les expériences "temps réel" et les "cinétiques rapides". "Temps réel" signifie un film de l'échantillon après une perturbation, par exemple un saut en température, une variation de $\mathrm{pH}$, une dilution, un mélange ou encore l'application d'un champ magnétique. Par "rapide" on entend des temps d'acquisition courts, de l'ordre de quelques centaines de millisecondes pour suivre les toutes premières étapes de formation et d'organisation de l'échantillon. La connaissance d'éventuelles phases intermédiaires peut être cruciale pour comprendre les propriétés de l'échantillon dans son état d'équilibre. Pour ces expériences, un appareil à flux stoppé (Bio-Logic $\left.{ }^{\mathrm{TM}}\right)$ a été spécialement adapté pour la DNPA. Il permet de mélanger rapidement deux à trois solutions en contrôlant précisement les volumes et les temps de mélanges puis de démarrer l'observation en connaissant le délai après le début de la réaction. Cette nouvelle technique a été utilisée pour suivre la croissance de vésicules formées après dilution de micelles d'AOT par une solution saline. Le moteur de la croissance est l'écrantage des répulsions électrostatiques entre les têtes polaires tensioactives, ce qui favorise la formation d'une bicouche localement plane.
\end{abstract}

\section{INSTRUMENTATION ET TECHNIQUE}

La nouvelle génération des diffractomètres de diffusion de neutrons aux petits angles, comme D22 à l'ILL ouvre de nouvelles perspectives avec les expériences "temps réel" et les "cinétiques rapides". Avec le large domaine en q couvert par une seule configuration $(\mathrm{qmax} / \mathrm{qmin}=20)$ et le flux élevé au niveau échantillon (jusqu'à $10^{8}$ neutrons $/ \mathrm{s} / \mathrm{cm}^{2}$ ), D22 offre d'uniques possibilités pour les expériences de cinétique [1].

L'intérêt d'un apparareil à flux stoppé est de contrôler le mélange de plusieurs solutions dans un temps court (entre 10 à $90 \mathrm{~ms}$ ) et de connaître précisément le temps entre la fin du mélange et le début de l'observation. La figure 1 décrit schématiquement le fonctionnement de l'appareil. Le SFM-3 (BioLogic) disponible sur D22 dispose de trois réservoirs de $20 \mathrm{ml}$ chacun, contrôlés par des moteurs pas à pas. Une première chambre de mélange se trouve en sortie des seringues 1 et 2 , suivie d'une ligne à retard et d'une seconde chambre de mélange. Le volume de la ligne à retard determine le temps de mûrissement $t_{\mathrm{ET}}$ entre les deux mélangeurs. La seconde chambre permet de mélanger la solution $(1+2)$ sortant de la ligne à retard avec la solution contenue dans la seringue 3 . La solution finale $(1+2+3)$ est stoppée dans la cellule d'observation. Par définition, le temps mort $t_{\mathrm{DT}}$ est le temps mis par la solution entre la seconde chambre de mélange et le milieu de la cellule d'observation. Le temps minimal pour démarrer l'osbservation $\left(\mathrm{t}_{\mathrm{DT}}+\mathrm{t}_{\mathrm{ET}}\right)$ est de l'ordre de 50 à $200 \mathrm{~ms}$ selon le débit. Lorsqu'on travaille avec uniquement deux solutions, il est judicieux d'utiliser les seringues 1 (ou 2 qui lui est équivalente) et 3. Le temps mort est réduit à $\mathrm{t}_{\mathrm{DT}}$.

La cellule d'observation est une cellule rectangulaire en quartz de $1 \mathrm{~mm}$ d'épaisseur, de $10 \mathrm{~mm}$ de largeur et $20 \mathrm{~mm}$ de hauteur ouverte aux deux extrémités. Un diaphragme de $6 \times 9 \mathrm{~mm}^{2}$ placé à la fin du guide de collimation définit la taille du faisceau direct à la position de l'échantillon. 


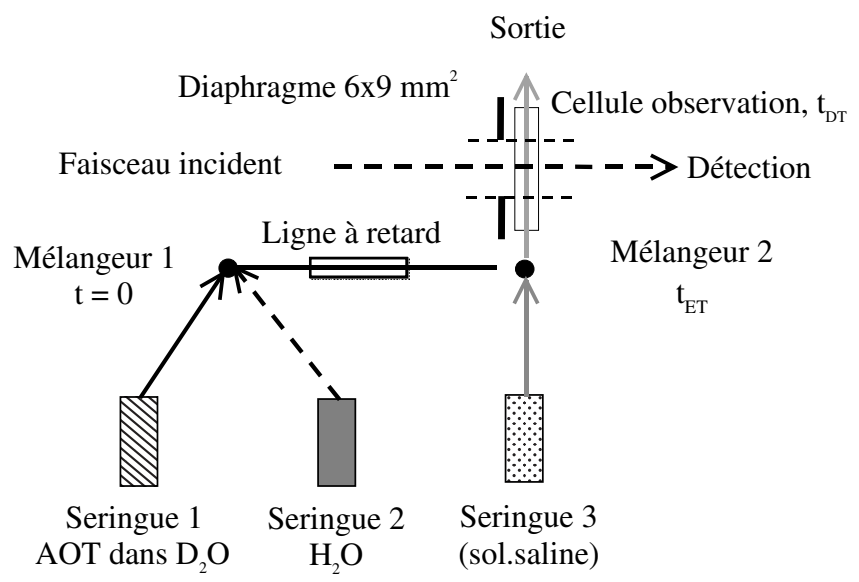

Figure 1. Fonctionnement du SFM-3.

La séquence de mélange définit les volumes et les temps de mélange. Le volume minimum est de $20 \mu$ l et le débit maximal de $2 \mathrm{ml} / \mathrm{s}$ pour éviter les turbulences dans la cellule. A cause de la géométrie de la cellule au moins $600 \mathrm{ml}$ de solution (soit 3 fois le volume de la cellule) sont nécessaires pour vider complètement la vieille solution et la remplacer par une fraîche.

Au moment choisi dans la séquence de mélange, l'électronique de l'appareil à flux stoppé peut envoyer un signal TTL $(0-5 \mathrm{~V})$ à l'ordinateur de contrôle du spectromètre qui démarre alors la séquence d'acquisition. La séquence d'acquisition définit le nombre de spectres et le temps de chacun. Pour une bonne précision de la mesure de temps, le temps en seconde est converti en une unité appelée tick (1 tick $=90.9 \mathrm{~ns})$. La carte électronique développée pour les expériences de cinétique peut stocker temporairement jusqu'à 450 spectres sans temps mort entre deux acquisitions successives. Le temps maximal par acquisition est de $390 \mathrm{~s}$.

Les séquences d'acquisition les plus utilisées suivent soit une série arithmétique $\left(t_{n}=a\right)$, soit une série géométrique ( $t_{n}=a^{n-1} t_{1}$ ), avec $t_{n}$ le temps d'acquisition du spectre numéro $n$ et $a$ la raison de la suite. La suite géométrique permet de bien suivre les tous premiers stages de la réaction. Il est également possible de définir le temps de chaque acquisition. Le temps minimal et "raisonnable" d'acquisition sur D22 est de l'ordre de 100 à 200 ms.

A la fin de la séquence d'acquisition, les données sont transférées vers la station de contrôle de l'instrument. Il est alors possible de répéter l'expérience pour additionner les spectres correspondant au même temps après mélange afin d'améliorer la statistique.

Le schéma de principe d'une expérience combinant DNPA et appareil à flux stoppé est résumé figure 2 .

\section{CROISSANCE DE VESICULES}

La première expérience réalisée sur D22 combinant le flux stoppé et la DNPA était consacrée au suivi de la transition entre micelles et vésicules et de leur croissance après addition de sel. Les vésicules peuvent être décrites comme des sphères creuses dont l'enveloppe est formée par une bicouche de molécules tensioactives. Utilisées comme modèle pour les membranes biologiques, elles trouvent d'autres applications comme microréacteurs ou pour l'encapsulation et la délivrance de substances actives.

\subsection{Matériel et méthodes}

Nous travaillons avec un tensioactif anionique le bis(2-ethyl hexyl)sulfosuccinate sodium, plus communément appelé AOT. Le diagramme de phase de l'AOT dans l'eau a été étudié depuis plus de 


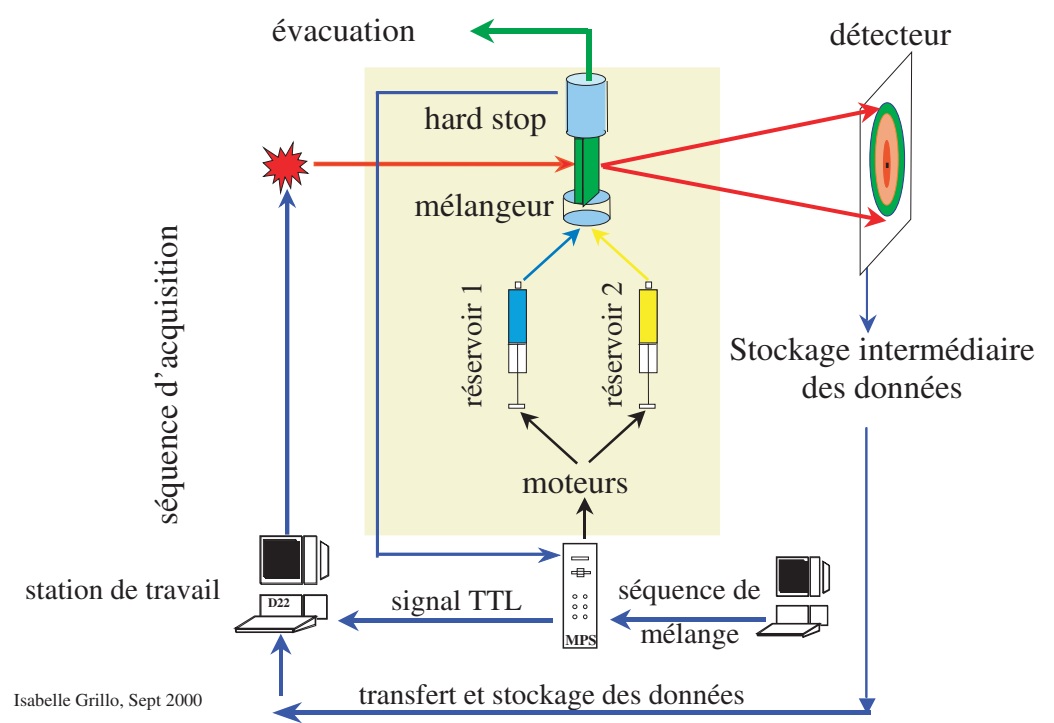

Figure 2. Schéma de principe d'une expérience combinant DNPA et "flux stoppé".

25 ans [2]. L'AOT forme des micelles entre la $\mathrm{cmc}(3,1 \mathrm{mmol} / \mathrm{l})$ et une fraction massique $\mathrm{w}=1,4 \%$. Entre 1,4\% et $10 \%$ les micelles sont en équilibre avec une phase lamellaire à son gonflement maximal. Pour des concentrations supérieures, on observe la formation d'un domaine monophasique lamellaire. La présence de vésicules n'est pas mentionnée dans les diagrammes de phases. En effet, comme nous le verrons dans la suite, les vésicules sont un état métastable qu'il n'a été possible de mettre en évidence que grâce à cette expérience de cinétique.

Le but cette étude est de comprendre l'effet de la nature du sel et de sa concentration sur la vitesse de croissance et la taille des vésicules. Nous avons travaillé avec 4 sels $(\mathrm{NaCl}, \mathrm{NaBr}, \mathrm{KCl}, \mathrm{KBr})$ et des concentrations de $0,08,0,17,0,37$ et $0,68 \mathrm{~mol} / \mathrm{l}$. La solution mère d'AOT est preparée dans $\mathrm{D}_{2} \mathrm{O}$ à une concentration massique de $1 \%$. Seuls des résultats avec $\mathrm{NaCl}$ seront présentés dans cet article. Létude complète est décrite dans [3].

Le premier réservoir contient la solution tensioactive, le réservoir 3, le solution saline. Le réservoir 2, rempli d'eau est utilisé pour rincer la cellule d'observation entre deux injections (figure 1). Nous mélangeons $900 \mu \mathrm{l}$ d'AOT en solution avec $300 \mu \mathrm{l}$ de solution saline pendant $600 \mathrm{~ms}$. Les compositions finales des échantilons sont $\mathrm{w}=0,75 \%$ d'AOT et $0,021,0,042,0,085$ et $0,17 \mathrm{~mol} / \mathrm{l} \mathrm{de} \mathrm{NaCl}$. L'acquisition est démarrée juste à la fin de la séquence de mélange, soit un temps mort $\mathrm{t}_{\mathrm{DT}}=90 \mathrm{~ms}$. Pendant les 10 premières minutes après le mélange, les temps d'acquisition suivent une série géométrique de raison 1,1 avec $\mathrm{t}_{1}=500 \mathrm{~ms}$. L'expérience est répétée 10 fois pour une bonne statistique. Entre $10 \mathrm{mn}$ et 1 heure, nous suivons la croissance par des acquisitions de $2 \mathrm{mn}$, puis des acquisitions de $3 \mathrm{mn}$ entre 1 heure et jusqu'à 5 heures après le début de la réaction.

La longueur d'onde est fixée à $6 \AA$ A. Pendant les 10 premières minutes après le mélange, nous utilisons une configuration "moyens angles", avec une distance entre l'échantillon et le détecteur de $\mathrm{D}=5 \mathrm{~m}$ et une collimation à $\mathrm{C}=5,6 \mathrm{~m}$ pour couvrir une gamme de vecteurs d'onde de $10^{-2}$ à $0,14 \AA^{-1}$. Pour les temps longs et pour suivre la croissance des objets, le détecteur est éloigné à $8 \mathrm{~m}$, avec une collimation à $8 \mathrm{~m}\left(610^{-3}\right.$ à $\left.8,710^{-2} \AA^{-1}\right)$.

L'évolution de l'intensité diffusée pendant les 10 premières minutes après le début de la réaction est présentée figure 3. L'oscillation est caractéristique du facteur de forme d'une sphère creuse et sa position est liée au rayon de la vésicule. Elle est visible dès la première acquisition soit $500 \mathrm{~ms}$ après le début de la réaction. La décroissance en $\mathrm{q}^{-2}$ visible dans une représentation double logarithmique est la signature 
d'objets localement plats. Le décalage de l'oscillation vers les petites valeurs du vecteur d'onde ainsi que l'augmentation de l'intensité aux petits q prouvent la croissance des vésicules.

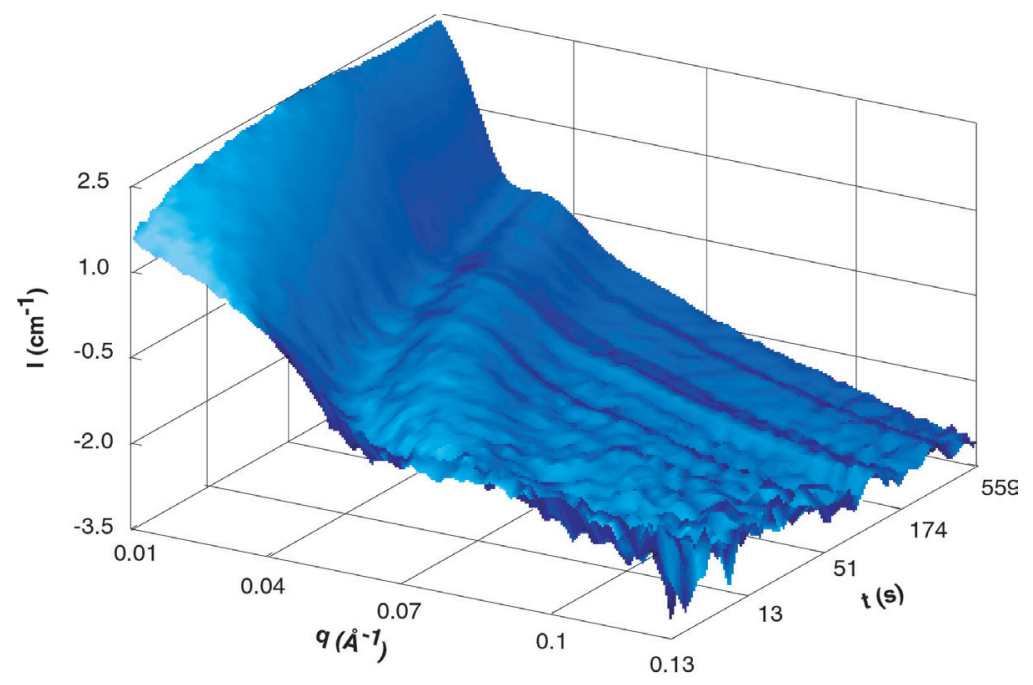

Figure 3. Evolution de l'intensité diffusée $\mathrm{I}\left(\mathrm{cm}^{-1}\right)$ pendant les 10 premières minutes après le début de la réaction. Composition finale de l'échantillon: AOT $\mathrm{w}=0,75 \%$ et $0,085 \mathrm{~mol} / \mathrm{l} \mathrm{NaCl}$.

\subsection{Modélisation de l'intensité diffusée}

L'intensité diffusée est modélisée par un modèle de sphère creuse [4].

$$
I(q)\left(\mathrm{cm}^{-1}\right)=\Phi V\left(\rho_{s}-\rho_{v}\right)^{2} F^{2}\left(q, r_{e x t}\right)=K_{c} F^{2}\left(q, r_{e x t}\right)
$$

$\Phi$ est la fraction volumique de tensioactif, $V$ le volume de la vésicule, $\rho_{s}$ et $\rho_{v}$ les densités de longueur de diffusion du solvant et de la bicouche d'AOT respectivement. $F$ est le facteur de forme et s'écrit:

$$
F\left(q, r_{e x t}\right)=\frac{3}{V_{e x t}-V_{i}}\left[V_{e x t} \frac{\sin \left(q r_{e x t}\right)-q r_{e x t} \cos \left(q r_{e x t}\right)}{\left(q r_{e x t}\right)^{3}}-V_{i} \frac{\sin \left(q r_{i}\right)-q r_{i} \cos \left(q r_{i}\right)}{\left(q r_{i}\right)^{3}}\right]
$$

$r_{i}$ et $r_{\text {ext }}$ sont les rayons intérieurs et extérieurs de la bicouche, $V_{i}$ et $V_{\text {ext }}$ sont les volumes intérieurs et extérieurs.

L'intensité (Eq. 1) est convoluée par une fonction log-nomale $G$ qui représente la polydispersité sur le rayon des vésicules puis par la fonction de résolution de l'instrument $R$, modélisée par une fonction gaussienne. Les détails de ce calcul se trouvent dans [5].

$$
I_{\text {model }}(q)=\int_{0}^{\infty} R\left(q, \Delta q, q^{\prime}\right) \int_{0}^{\infty} K_{c} G\left(r_{e x t}, \sigma, r^{\prime}\right) F^{2}\left(r^{\prime}, q^{\prime}\right) d r^{\prime} d q^{\prime}
$$

L'évolution du rayon des vésicules en fonction du temps est préSentée sur la figure 4 pour deux concentrations en sel $(0,021$ et $0,085 \mathrm{~mol} / 1 \mathrm{de} \mathrm{NaCl})$. Nous observons une diminution de la vitesse de croissance lorsque la force ionique augmente. Avec le temps la polydispersité des vésicules augmente fortement. L'oscillation du facteur de forme disparaît et seul reste une décroissance en $\mathrm{q}^{-2}$, caractéristique d'objets plats et il n'est plus possible d'utiliser l'équation 1. Les vésicules sont dans un état métastable et le système évolue vers des bicouches ouvertes en équilibre avec des micelles. 


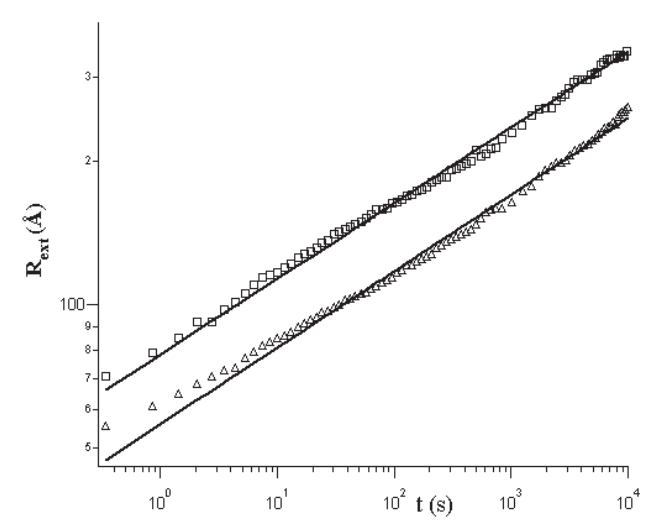

Figure 4. Evolution du rayon des vésicules en fonction du temps. Composition finale: AOT w $=0,75 \%$ (i) $\mathrm{NaCl}$ $0,021 \mathrm{~mol} / 1(\Delta) 0,085 \mathrm{~mol} / 1$. Les lignes correspondent au meilleur paramétrage selon l'équation 8 .

\subsection{Modèle cinétique}

Quel est le moteur de la formation et de la croissance des vésicules? Le modèle suivant a été développé par E. Kats et A. Muratov [5] et implique un mécanisme en deux étapes. Les ions libres provenant de la dissociation des molécules du sel écrantent les répulsions électrostatiques entre les micelles ce qui permet leur agrégation. En parallèle, le sel écrante les interactions électrostatiques entre les têtes polaires tensioactives ce qui diminue la surface par tête et augmente le paramètre d'empilement de 0.33 (sphère) à 1 (plan). A la fin de cette première étape, relativement rapide $(<500 \mathrm{~ms})$ un certain nombre de vésicules relativement petites $\left(\mathrm{R}_{\text {ext }} \approx 50-70 \AA ̊\right)$ sont formées. Ensuite les vésicules grossissent par diffusion et incorporation de micelles dans la bicouche.

La variation du nombre d'agrégation $\mathrm{N}$ et fonction du temps est proportionnelle à la variation de l'énergie de courbure par rapport au nombre d'agrégation:

$$
\frac{d N}{d t} \propto \frac{d E_{N}}{d N}
$$

La contribution harmonique à l'énergie de courbure calculée par Helfrich [6] $E_{0}=8 \pi \kappa+4 \pi \bar{\kappa}$ est indépendante du nombre d'agrégation ( $\kappa$ est la rigidité de courbure et $\bar{\kappa}$ le module de courbure gaussien). Il faut considérer les contributions anharmoniques qui elles dépendent du nombre d'agrégation [7]:

$$
E_{a n}=4 \pi \frac{\kappa_{a n}}{R^{2}} \propto \frac{\kappa_{a n}}{a_{h} N}
$$

$\kappa_{\mathrm{an}}$ est un module anharmonique élastique. Après dérivation de l'équation 4, l'équation 3 devient:

$$
\frac{d N}{d t} \propto \frac{\kappa_{a n}}{R^{2}} \frac{a_{h}}{R^{2}}
$$

D'autre part, comme le nombre d'agrégation $\mathrm{N}$ est proportionnel au carré du rayon:

$$
d N / d t \propto R d R / d t
$$

En combinant les équations (4) et (5) on obtient finalement:

$$
R=A t^{1 / 6}=A t^{0.166}
$$

Pour les quatre concentrations en $\mathrm{NaCl}$ de $0,021,0,042,0,085$ et $0,17 \mathrm{~mol} / 1$, nous trouvons des exposants de $0,159,0,154,0,160$ et 0,159 respectivement, en bon accord avec le modèle cinétique proposé. 


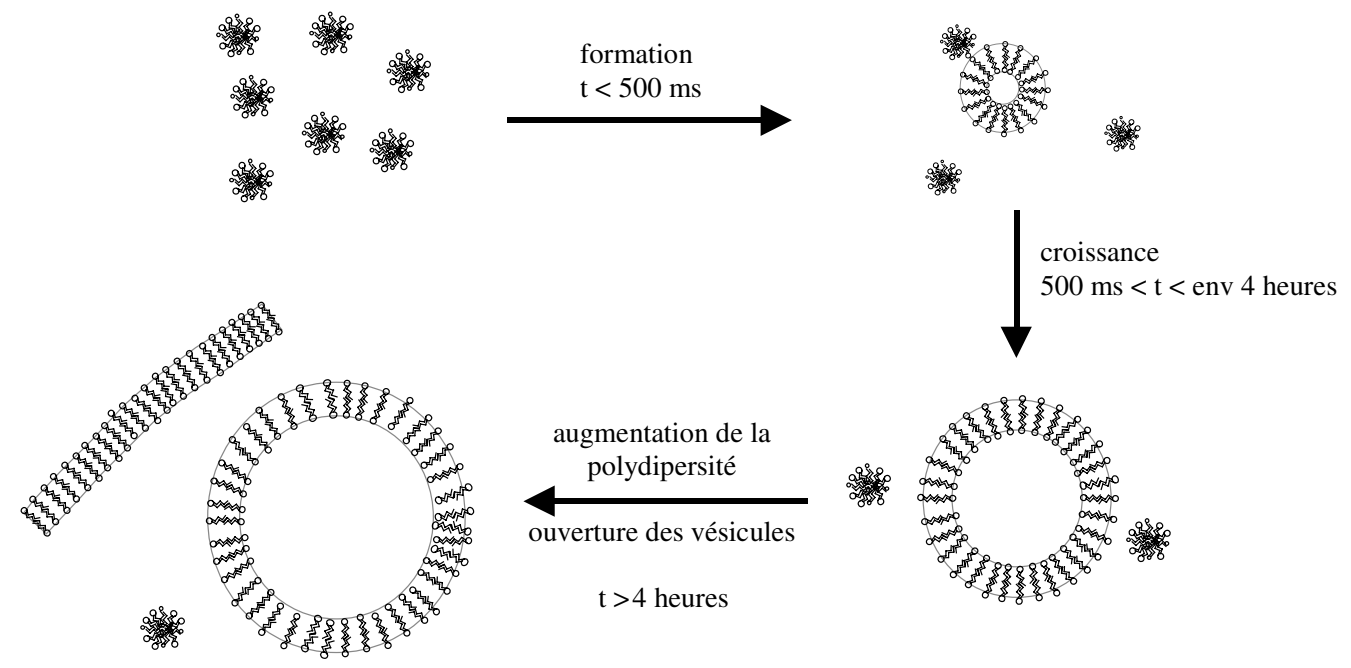

Figure 5. Représentation schématique du mécanisme de croissance des vésicules d'AOT.

\section{CONCLUSION}

Cette étude en cinétique a permis de mettre en évidence la présence de vésicules dans le diagramme de phases $\mathrm{AOT} / \mathrm{D}_{2} \mathrm{O}+\mathrm{NaCl}$, phase qui n'est mentionnée dans aucuns des diagrammes de phases disponibles dans la litérature. Les vésicules se forment dans un temps court, inférieur à $500 \mathrm{~ms}$. Le moteur de leur formation est l'écrantage par le sel des répulsions électrostatiques entre les têtes polaires adjascentes dans les micelles et entre les micelles. Les vésicules grossissent par incorporation des micelles dans leur bicouche pendant plusieurs heures. Ces vésicules sont dans un état métastable et elles évoluent après 4 ou 5 heures vers un équilibre entre des micelles et des bicouches, précurseurs de la phase lamellaire formée à plus haute concentration (figure 5).

La connaissance des lois de croissance est essentielle pour la formulation. Par exemple, elles permettent de connaître le temps auquel il faut stopper une réaction pour obtenir des particules de taille voulue. De leur comportement, il est également possible de comprendre les mécanismes de formation et d'évolution de l'échantillon.

\section{Références}

[1] http://www.ill.fr/YellowBook/D22/ and http://www.ill.fr/YellowBook/D22/D22_info/

[2] Skouri M., Marignan J., May R., Colloid. Polym. Sci 269 (1991) 929; Balinov B., Olsson U., Söderman O., J. Phys. Chem. 95 (1991) 5931; Frances E. I., Hart T. J., J. Colloid and Interf. Sc. 94 (1983) 1.

[3] Grillo I., Kats E.I., Muratov A.R., Langmuir 19 (2003) 4573.

[4] Pedersen J.S., Adv. Colloid Interface Sci. 70 (1997) 171.

[5] Grillo I., Effect of instrumental resolution and polydispersity on ideal form factor in Small Angle Neutron Scattering 2000, ILL Technical Report n ${ }^{\circ}$ ILL01GR08T.

[6] Helfrich W.Z., Naturforsch. 28c (1973) 693.

[7] Helfrich W.Z., J. Phys. 47 (1986) 321. 